\title{
Los sistemas de gestión electrónica de la documentación y la teoría del ciclo vital de los documentos en las organizaciones
}

\author{
Carlota Bustelo Ruesta. \\ Inforárea S.L.
}

\subsection{Resumen}

La utilización de tecnologías informáticas para la creación de documentos y la gestión electrónica de la documentación obliga a replantearse la actuación de los especialistas en información y documentación. En el plano teórico, se proponen cambios en el concepto de documento y archivo, y en la teoría del ciclo vital de los documentos. En el plano práctico se proponen estrategias para la preservación y gestión de los documentos electrónicos. (Autor)

Palabras clave: Documentos electrónicos. Gestión de documentos. Gestión electrónica de la documentación.

\subsection{Abstract}

The usage of information technologies in the record creation and document electronic management forces a reformulation of the performance of information and documentation specialist. On the theorical level, changes are proposed in the concept of record and archive and in the theory of life cycle of records. On the practical level, strategies are proposed for the preservation and management of the electronic documents. (Autor)

Keywords: Electronic documents. Record management. Document electronic management.

\section{Introducción}

La rápida evolución de las tecnologías de la información, que nos obliga a asimilar novedades a gran velocidad, ha permitido la aparición de productos y herramientas informáticas para la gestión electrónica de la documentación. Este concepto, cuya última finalidad es conseguir la "oficina sin papeles", está teniendo una gran aceptación entre los directivos de las organizaciones, obligados a 
planificar estrategias para actuar con mayor eficacia en un mundo cada vez más competitivo.

Cuando tratamos de aproximarnos a como se deben implantar estas tecnologías nos encontramos con una realidad de dos caras completamente diferentes:

Por un lado, el mundo de la tecnología en el que se manejan con soltura conceptos como documento electrónico, e-mail, formato EDI, Intranets, etc. Y que está dominado por especialistas en tecnologías de la información, habitualmente informáticos o ingenieros de telecomunicaciones.

Por otro lado, tenemos el mundo de la formulación teórica y práctica de la gestión de documentos (records management) o la archivística moderna, basada en la existencia del ciclo vital de los documentos que han desarrollado los grandes archiveros.

Parece obvio que deberían ser dos mundos complementarios a la hora de implantar sistemas de gestión electrónica de documentos; pero una cierta incomprensión mutua y la falta de dialogo los separan cada vez más. Sorprende que en el desarrollo y la implantación de sistemas de gestión documental no se cuente, en la mayor parte de los casos, con especialistas en gestión documental. Pero es igual de sorprendente encontrar que se siguen publicando manuales de gestión de la documentación que abordan la gestión electrónica de documentos de una forma tangencial resolviéndolo en un capítulo habitualmente llamado "Nuevas tecnologías".

Mientras tanto en la realidad de las organizaciones se deben tomar decisiones que afectan a la gestión documental y a la implantación de nuevas tecnologías. Actualmente puede afirmarse que en casi todas las organizaciones los documentos se producen electrónicamente por medio de los paquetes ofimáticos instalados en microordenadores. En menor medida, pero imponiéndose a gran velocidad los documentos se trasmiten también por medios electrónicos de forma que no deben pasar obligatoriamente por la forma papel. Los hábitos de la gestión documental no han sabido adaptarse a los nuevos soportes y medios y se ha producido una dejadez en el control de los documentos en papel, que no ha sido sustituido por el control de los documentos electrónicos. Algunos ejemplos de lo que está ocurriendo podrían ser:

- Archivos de oficina repletos de copias en papel de documentos electrónicos, que también están almacenados en el disco duro del ordenador. Para poder encontrarlos se debe buscar la copia en papel, con todas las limitaciones de un sistema manual, y si se ha tenido la precaución de anotar a lápiz el nombre del fichero y directorio electrónico donde se encuentra el documentos se podrá recuperar el documento electrónico. 
- Archivos de organizaciones que guardan estanterías enteras de disquetes y cintas sin ninguna utilidad, ya que no existen máquinas capaces de leerlos

- Documentos producidos por un procesador de textos, que son impresos para luego poder escanearlos e introducirlos en el sistema de gestión documental.

Frente a esta realidad, los especialistas en información y documentación debemos actuar en dos planos diferentes. Por un lado, es necesario reformular las bases teóricas de la gestión documental, de forma que amplíen y adapten los conceptos a la realidad actual. Por otro, necesitamos estrategias prácticas que resuelvan los problemas cotidianos que supone la gestión de documentos electrónicos y la implantación de sistemas de gestión electrónica de la documentación.

Esta doble preocupación es compartida por profesionales de todos los países y se encuentra perfectamente expresada en los documentos del Comité sobre Documentos Electrónicos del Consejo Internacional de Archivos (Guide...., 1996). Otro buen ejemplo se encuentra en las páginas puestas en Internet por los Archivos Australianos: Guidelines for Managing Electronic Documentns in Australian Government (1995)

Esta comunicación es una reflexión sobre algunos aspectos teóricos y prácticos surgidos de la experiencia en la implantación de sistemas de gestión de la documentación.

\section{Las bases teóricas}

Todos los cambios tecnológicos han planteado la necesidad de una nueva teoría de la información, que entre otras cosas, amplíe y adapte los conceptos de gestión documental y ciclo vital de los documentos. La última gran formulación teórica de Schellenberg $(1956,1965)$ sigue siendo repetida prácticamente al dedillo en todos los manuales al uso. Aunque desde hace mucho tiempo se tiene conciencia de que "las tecnologías de la información van a permitir expandir el campo teórico en el contexto de la teoría de la información" (Cook, 1988, p. 20), todavía estamos esperando la formulación definitiva. Esta teoría en cuanto a la gestión documental deberá incidir al menos en tres puntos claves:

\subsection{El concepto de documento}

Ya desde hace tiempo las definiciones de documento se hacen de una forma amplia para que además de documentos en papel puedan incluir cualquier otro tipo de soporte. Se dice que un documento es la combinación de un soporte y la información registrada en él, que puede ser utilizado como prueba o para consulta. Siguiendo esta definición nuestros centros de documentación y bibliotecas han empezado a recoger todo tipo de materiales como CD-ROM, vídeos, regis-

Scire. 3: 2 (jul.-dic. 1997) 45-53 
tros sonoros, etc., incorporándolos sin excesivos problemas a sus sistemas de información.

Sin embargo, tenemos más problemas de aplicación en los documentos originales relacionados con una actividad llevada a cabo por un individuo o entidad, tradicionalmente considerados de archivo. Es obvio que este tipo de documentos se generan en la actualidad en un alto porcentaje por medios electrónicos (procesadores de texto, e-mail, formato EDI, etc.). Para que estos documentos no se queden fuera del sistema definimos un documento como la evidencia de un acontecimiento registrada sobre un soporte concreto, que permite su recuperación posterior; pero todas las características que atribuimos a los documentos están totalmente basadas en los documentos en papel. Y es que los documentos electrónicos tienen unas características especiales que cambian mucho el concepto de documento:

- Son legibles por máquinas no por personas, por lo que para poder hacer efectiva la recuperación posterior es necesario tener la máquina adecuada. ¿Son por lo tanto las máquinas parte esencial de los documentos?

- El contenido de un documento electrónico no esta ligado para siempre a su soporte. En un documento en papel nunca podemos desligar el contenido del soporte; en un documento electrónico no sólo podemos hacerlo, sino que debemos hacerlo en muchos casos si queremos que sigan siendo legibles. Pasaremos de un disco duro a otro al cambiar de ordenador, de disquetes de 5 y cuarto a disquetes de 3 y medio, etc.

- Profundizando en la característica anterior, en los documentos electrónicos la estructura física carece de importancia ya que no permanece inalterable. El papel que cumple la estructura física en los documentos en papel es sustituida por la estructura lógica de los documentos electrónicos, que hace posible identificar el documento y representar sus estructuras internas. Es el nombre del documento y su extensión, la forma que están dispuestos los datos, etc.

- Los documentos electrónicos necesitan además de unos "metadatos" sobre su contexto y estructura que permiten hacerlos entendibles y usables. Entre estos están la fecha en que se creo el documento, el programa que lo creó, el autor del mismo, etc.

\subsection{El ciclo vital de los documentos}

Desde hace tiempo la gestión de documentos se concibe como el desarrollo de un sistema organizado para la creación, almacenamiento y acceso a los documentos producidos en el contexto de una determinada organización. En este contexto todos los documentos siguen un ciclo vital que podría resumirse en las 
siguientes fases: creación, uso, mantenimiento y eliminación/conservación. La intervención de especialistas en la gestión documental se ha realizado sobre todo en las últimas etapas de vida de los documentos, teniendo menos influencia en la etapa de creación. Con los documentos electrónicos esta primera etapa cobra cada vez más importancia, pudiéndose hablar de una etapa todavía anterior de gran importancia: la concepción del sistema documental. A cada persona o función dentro de la organización se le deben asignar las herramientas adecuadas y los derechos para que pueda crear los documentos oportunos. El sistema establecido debe asegurar al menos:

- que los documentos electrónicos creados pueden mantenerse accesibles y legibles a lo largo del tiempo.

- que los documentos electrónicos, mediante su contenido, contexto y estructura den evidencia fidedigna de las actividades de su creador, salvaguardándolos de las posibles manipulaciones y alteraciones.

- que los documentos vitales han sido identificados y se ha establecido cual es la mejor forma para conservarlos.

\subsection{EI archivo virtual}

La etapa de mantenimiento y conservación de los documentos ha estado tradicionalmente ligada con la gestión física de los mismos, identificándose el sistema de archivo con el espacio físico dónde se reunían los documentos. En una organización sin papeles nunca puede desaparecer el archivo, pero pasa de ser una realidad física a una realidad virtual. Aunque los documentos residan en distintos ordenadores o dispositivos lógicos que físicamente estén separados, los documentos que dan fe de la actuación de una organización no tienen sentido aisladamente, por lo que no deben perder la unidad necesaria para su comprensión.

\section{Las estrategias prácticas}

Sin embargo, en la resolución de los problemas del día a día no podemos esperar a la teoría integradora de la gestión documental y de la información. La creación de documentos en soportes diferentes que el papel es una realidad cotidiana que se extiende de forma irrefrenable, la aplicación de sofisticados sistemas de gestión electrónica de la documentación se impone en las empresas más punteras y ejemplos de comunicación a través de las redes para la solicitud o envío de información son cada vez más frecuentes. Por ello, es necesario establecer como profesionales de la información y la documentación una serie de estrategias prácticas que nos permitan gestionar los sistemas de gestión documental en los que intervienen documentos diferentes al papel. Sin pretender dar recetas mágicas, nuestra experiencia hace destacar los siguientes puntos:

Scire. 3: 2 (jul.-dic. 1997) 45-53 


\subsection{Participar en la implantación de las tecnologías}

La participación de expertos en documentación e información en los procesos de implantación de tecnologías es el primer paso a conseguir. Muchas veces estos procesos sirven de excusa y motivo para plantear un buen sistema de gestión de la documentación. En estos proyectos la colaboración con otros profesionales (informáticos, especialistas en gestión, etc.) es imprescindible, debiéndoles convencer de la importancia que tienen los requerimientos exigidos al sistema por la gestión documental. Habitualmente un informático no se plantea la conservación de los documentos vitales, ni la unidad del archivo de documentos como reflejo de la actividad de una organización, y aunque muchas veces se plantean la seguridad de los sistemas y la asignación de derechos no ven en esto ninguna implicación documental.

La intervención en estos procesos iniciales nos permitirá una acción desde las primeras etapas del ciclo vital de los documentos, que siempre resulta mucho más efectiva que cuando sólo nos dejan intervenir en las etapas finales.

Además estos proyectos suelen contar con la involucración del personal afectado y el apoyo de la alta dirección dentro de la organización, lo cual nos ofrece una oportunidad importante para, por un lado, conocer a fondo las necesidades que debe cubrir un buen sistema de gestión documental y, por otro, hacer "marketing" de la importancia de los servicios que prestamos como profesionales.

\subsection{Formación en tecnología}

Actualmente ningún profesional de la información y la documentación puede desligar el ejercicio de su profesión de la utilización de herramientas tecnológicas de informática y telecomunicaciones. Sin pretender sustituir a informáticos e ingenieros en sus tareas específicas, el conocimiento a fondo de estos instrumentos es básico para la formulación de un buen sistema de gestión documental.

Esta formación debe estar compuesta al menos por tres componentes: la comprensión de cómo funcionan las herramientas, la destreza en el manejo de las mismas (cuando se ha manejado uno o dos sistemas es fácil utilizar otro) y el conocimiento de las novedades que día a día van apareciendo y que pueden ayudar a mejorar nuestro sistema.

Esta autoformación, que requiere en muchos casos un gran esfuerzo personal, es indispensable si queremos intervenir en los procesos de implantación de sistemas de gestión documental.

\subsection{Claves para el día a día}

Mientras pensamos como sería un buen sistema o decidimos la tecnología adecuada o la dirección de la organización decide acometer el proyecto en serio, en todas las organizaciones se están produciendo documentos electrónicos que se 
almacenan en los discos duros o ópticos probablemente sin el menor control. A veces cuando nos piden que intervengamos (sobre todo ejerciendo la labor de consultoría) el caos es tal, que deshacerlo resulta un esfuerzo titánico y a veces imposible. Algunas pequeñas medidas pueden ayudar a que esta situación se haga más abordable:

3.3.1. En los cambios de sistema informático hay que tener en cuenta siempre cual va a ser nuestra opción de preservación de los documentos que se han realizado con la tecnología que se sustituye. No existen modelos únicos aplicables a todos los casos, pero pueden distinguirse tres tendencias o posibilidades:

- Preservar la tecnología con que fueron creados los documentos, tanto en hardware como en software para que estos documentos puedan ser leídos siempre. Esta opción causa problemas importantes pues no es fácil mantener tecnologías obsoletas que nadie sabe arreglar y además podría convertir a los archivos del futuro en una especie de Museos de la Informática. Sin embargo, la idea de un centro de preservación de tecnologías antiguas que permitan reutilizar los documentos o reconvertirlos para su uso posterior se va haciendo eco entre las instituciones y profesionales que han abordado este problema.

- Eliminar la dependencia de una tecnología concreta exigiendo la utilización de estándares que puedan ser utilizados desde distintos sistemas. Esta idea que siempre nos vende nuestro proveedor de informática se ha ido imponiendo en los últimos años creándose estándares para aplicaciones determinadas y estándares de facto como la filosofía de sistemas abiertos "Microsoft"; sin embargo tenemos por un lado los documentos producidos con sistemas más antiguos y por otro la constatación de que en la práctica los estándares no son tan compatibles como en la teoría.

- La tecnología nos presenta también soluciones intermedias que se incluyen en muchas aplicaciones. Son los denominados "visualizadores" o programas que nos permiten visualizar y por lo tanto leer documentos realizados con otras aplicaciones sin necesidad de tener los programas. O los programas que convierten en imágenes documentos realizados con distintas aplicaciones y que a partir de entonces pueden consultarse como cualquier otra imagen. Estas tecnologías tienen importantes implicaciones en la autenticidad de los documentos, pues al no poder ser editados con la herramienta original las modificaciones a posteriori (preocupación fundamental a la hora de conceder validez a los documentos electrónicos) son mucho más difíciles de realizar. Estas aplicaciones las estamos utilizando en diversos contextos: cuando mandamos un fax por una tarjeta de fax/módem, cuando visualizamos anexos de un correo electrónico, etc. Las limitaciones que tie-

Scire. 3: 2 (jul.-dic. 1997) 45-53 
nen es que no pueden ser universales, aunque las capacidades que incluyen son las que utilizamos todos más habitualmente.

3.3.2. Para ejercer el control intelectual sobre los documentos electrónicos además de utilizar las mismas fórmulas que utilizamos para los documentos en papel: cuadro de clasificación, descripción del contexto en que se producen y control de su localización física, debemos tener en cuenta nuevos datos muy importantes. Estos datos partirán de las reglas para su creación, modificación y borrado, en que aplicaciónes se han realizado, cual es el formato o códigos con que se han construido, cual es el sistema de nomenclatura de los mismos, etc.

3.3.3. La organización lógica de unidades de almacenamiento de documentos en los discos duros o discos ópticos puede tener mucha importancia al plantear un sistema de gestión documental. Conociendo las distintas etapas del ciclo vital de los documentos los subdirectorios o unidades en las que se almacenan los documentos deberían estar compuestos por documentos que tienen las mismas características en su paso a distintas etapas. Por ejemplo, en una organización que han decidido expurgar sus albaranes pasados dos años, llevarlo a cabo es más fácil si se han guardado todos en un mismo disco óptico, que si se encuentran mezclados con las facturas que deben guardarse hasta cinco años.

\section{A modo de conclusión}

En este mundo cambiante, nos pasamos gran parte de nuestro tiempo imaginando como serán las cosas en el futuro al mismo tiempo que tratamos de asimilar las novedades del presente. En la esfera profesional de la gestión de la documentación y la información la sensación de vértigo es mucho más fuerte y quizás por ello cada uno de los temas que tocamos nos incita a reflexionar sobre el futuro profesional, la capacidad de afrontar los retos que se nos presentan y el papel que debemos jugar como profesionales. Sin abandonar este sano ejercicio, deberíamos además empezar a discutir, compartir experiencias, contrastar opiniones sobre temas como el de esta comunicación.

\section{Referencias}

Badillo Nieto, Margarita. (1995). La gestión de documentos electrónicos : ¿asignatura pendiente? // Base. 26 (1995) 37-41

Broadhurst, Roger N.(1993). Document Management Yearbook 1994. A guide to imaging and document management products and services. Hertfordshire : Cimtech Limited, 1993

Bustelo Ruesta, Carlota (1994). El papel de los expertos en documentación en la implantación de tecnologías de gestión de documentos. // IV Jornadas Españolas de Documentación Automatizada. Oviedo : Universidad, 1994. 357-362

Bustelo Ruesta, Carlota. La aplicación de un sistema de gestión de la documentación en

Scire. $3: 2$ (jul.-dic. 1997) 45-53 
una empresa de servicios : estudio de un caso. // 5es. Jornades Catalanes de Documentació. 369-379

Codina, Lluis (1994). Sistemas de gestión documentales : estado del arte y estrategias de utilización. // Binary. 62 (1994) 114-119

Codina, Lluis (1992). Gestión electrónica de documentos : basados en microordenadores de alto nivel y en memorias ópticas... // Binary (1992) 63-75

Codina, Lluis (1994). Sistemas ofimáticos de gestión documental : Lotus Notes. // Information World en Español. 27 (1994)

Cook, Michael (1988). Information Technology: A challange to training. // Archivium. 35 (1988)

Garcia Morales Huidobro, Elisa (1995). Del archivo en papel a los sistemas de gestión electrónica de documentos. // Information World en Español. 36 (1995) 20-22

Guide for managing electronic records from an archival perspective: Consultation draft. International Council on Archives: 1996. Committee on electronic Records. June 1996. URL : http://www.archives.ca/ica

Guidelines for Managing Electronic Documents in Australian Goverment Agencies (1995). Commonwealth of Australia, 1995. URL : http://www.aa.gov.au

Green, William B.(1993). Introduction to electronic document management systems. Boston [etc.] : Academic Press, cop. 1993

Kesner, Richard M. (1993). El paradigma de la gestió dels nous recursos d'informació i les seves implicacions per als arxivers i els gestors de documents. // LLIGALL : Revista catalana d'arxivistica. 6 (1993) 27-42

Llanso I Sanjuan, Joaquim (1993). Gestión de documentos : definición y análisis de modelos. Bergara: Irargi, 1993

Perpinya i Morera, Remei. El sistema documental de l'empresa : el punt de vista de l'arxiu i el centre de documentació. // 5es. Jornades Catalanes de Documentació. 337-344

Roberge, Michel (1965). La gestió dels documents administratius. Barcelona : Associació d'Arxivers de Catalunya, 1993.

Shellenberg, Theodore R. (1965). The management of Archives. New york : Columbia University press, 1965.

Shellenberg, Theodore R. (1956). Modern Archives: Principles and Techniques. Chicago : University of Chicago Press, 1956.

Scire. 3: 2 (jul.-dic. 1997) 45-53 